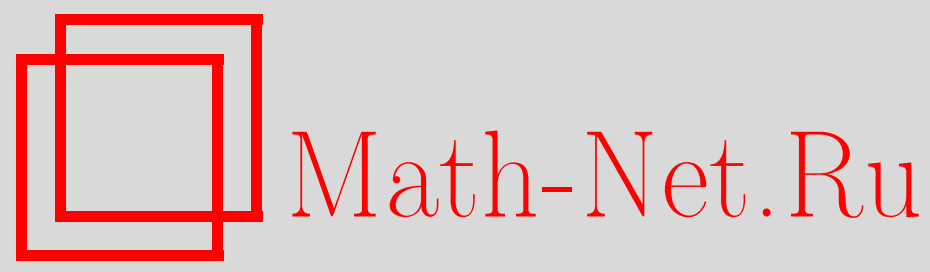

И. Ю. Фёдоров, Чисто логтерминальные раздутия индекса 1, Матем. заметки, 2004, том 75, выпуск 6, 917-926

DOI: https://doi.org/10.4213/mzm72

Использование Общероссийского математического портала Math-Net.Ru подразумевает, что вы прочитали и согласны с пользовательским соглашением http://www. mathnet.ru/rus/agreement

Параметры загрузки:

IP: 52.87 .193 .239

26 апреля 2023 г., 16:42:41

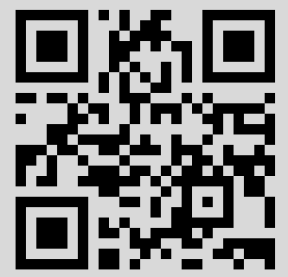




\title{
ЧИСТО ЛОГТЕРМИНАЛЬНЫЕ РАЗДУТИЯ ИНДЕКСА 1
}

\author{
И. Ю. Фёдоров
}

\begin{abstract}
В статье классифицированы чисто логтерминальные раздутия индекса 1 трехмерных терминальных особенностей.

Библиографоия: 17 названий.
\end{abstract}

Классификация особенностей, возникающих в лог-программеминимальных моделей, является одной из основных и наиболее трудных проблем современной бирациональной геометрии. Основные трудности начинаются уже в размерности 3 .

Хотя трехмерные терминальные особенности уже описаны (см. [1]), структуры их разрешений и описания исключительных дивизоров до сих пор не известны, за исключением некоторых частных случаев (см., например, [2]-[5]). В размерности 4 и вьше, вообще до конца не понятно, какие классы особенностей рассматривать.

Другой подход к классификации особенностей состоит в описании чисто логтерминальных раздутий особенностей и классификации реализуемых дивизоров. Этот подход тесно связан с теорией дополнений Шокурова [6], в которой особенности делятся на два класса: исключительные (в этом случае чисто логтерминальное раздутие единственно) и неисключительные (когда в линейной системе $n K$ содержится “хороший” дивизор для небольших $n$ ). Эти идеи используются в подходе Шокурова [7] к классификации флипов, где существенным шагом является индукция по размерности задачи. Подобную индукцию можно осуществлять, делая чисто логтерминальные раздутия и исследуя проблему уже на исключительном дивизоре (отметим, что в размерности $\leqslant 3$ чисто логтерминальные раздутия существуют для всех нормальных многообразий с логтерминальньгии особенностями [8], [9]).

В данной работе классифицированы чисто логтерминальные раздутия индекса 1 (т.е. исключительньй дивизор - дивизор Картье) терминальных точек, т.е. доказана

ТЕОРема 0.1. Чисто логтерминальные раздутия индекса 1 трехмерных терминальных особенностей таковы:

(1) $\left(\mathbb{P}^{2}, \mathbb{C}^{3},(1,1,1)\right)$ - гладкий случай;

(2) $\left(\left(t y+z^{2}+x^{2}=0\right) \subset \mathbb{P}^{3},\left(t y+z^{2}+x^{2}=0\right) \subset \mathbb{C}^{4},(1,1,1,1)\right)$;

(3) $\left(\left(t y+z^{2}=0\right) \subset \mathbb{P}^{3},\left(t y+z^{2}+x^{n}=0\right) \subset \mathbb{C}^{4},(1,1,1,1)\right)-$ cлучай $n>2$;

Работа выполнена при частичной поддержке Российского фонда фундаменталных исследований, грант № 02-01-00441, программы "Ведущие научные шшолы”, гранты № 00-15-96085 и № MK602.2003.01, и фонда INTAS-OPEN 2000, грант № 269. 
(4) $\left(\mathbb{P}^{2}, \frac{1}{2}(1,1,1),(1,1,1)\right)$;

(5) раздутие пучка идеалов приведенной неприводимой кривой в $\mathbb{C}^{3}$, которая локально представляется как полное пересечение.

Здесь представлены тройки $(S, Z, f)$, әде $S$ - исключительная поверхность, $Z$ уравнение особенности, $f$ - морфизм. Так как $f$ будет всегда взвешенным раздутием, мы будем писать веса этого раздутия.

Автор очень признателен В. А. Исковских и Ю. Г. Прохорову за плодотворные беседы и внимание к работе.

\section{1. Основные определения и предварительные результаты}

Все многообразия определены над $\mathbb{C}$. Все основные определения приведены в [10], [8] и [4].

ОПРЕДЕЛЕниЕ 1.1. Многообразие $X$ назьвается $\mathbb{Q}-$ факториальнылм, если некоторая целая кратность любого дивизора Вейля есть дивизор Картье. Многообразие $X$ назьвается $\mathbb{Q}$-горенштейновым, если некоторая кратность $K_{X}$ - дивизор Картье.

ОПРЕДЕЛЕНИЕ 1.2. Нормальное $\mathbb{Q}$-горенштейново многообразие $X$ uмеет не $x y$ же, чем терминальные (канонические, логтерминальные, логканонические) особенности, если для любого разрешения $f: Y \rightarrow X$ такого, что

$$
K_{Y}=f^{*} K_{X}+\sum a_{i} E_{i}
$$

все $a_{i}>0$ (соответственно $\left.a_{i} \geqslant 0, a_{i}>-1, a_{i} \geqslant-1\right)$.

Теорема 1.3 (Классификация терминальных особенностей). Пусть $(X, P)-$ росток трехмерной терминальной особенности.

1) Если индекс $(X, P)$ равен 1, то $(X, P)$ формально әквивалентно гиперповерхностной особенности $(\{\varphi=0\}, 0)$, где $\varphi$ принадлежит одной из следуюших серий:

(a) $\varphi=t y+f(z, x)$;

(b) $\varphi=t^{2}+f(y, z, x)$, әде $j_{2} f=0 u f_{3}(y, z, x)$ не делится на квадрат линейной $\oint о p \mathcal{M b l}$;

(c) $\varphi=t^{2}+y z^{2}+f(y, z, x)$, əде $j_{3} f=0$;

(d) $\varphi=t^{2}+y^{3}+f(y, z, x)$, где $j_{3} f=0 u j_{5} f=f_{4}+f_{5}$ содержит один из мономов

$$
y^{4}, z x^{3}, z^{2} x^{2}, x^{5}, z x^{4}, z^{2} x^{3}, y x^{3}, y z x^{2}
$$

с ненулевым коэффициентом.

2) Если индекс $(X, P) \geqslant 2$, то $(X, P)$ допускает одно из перечисленных ниже вложений:

(a) $X \simeq\{t y+f(z, x)=0\} / \mathbb{Z}_{m}(\alpha,-\alpha, 1,0)$, где $f(z, x)-\mathbb{Z}_{m}$-инвариант;

(b) $X \simeq\left\{t^{2}+y^{2}+f(z, x)=0\right\} / \mathbb{Z}_{4}(1,3,1,2)$, где $f(z, x)$ не содерэит $x$ иявляется $\mathbb{Z}_{4}$-инвариантом;

(c) $X \simeq\left\{t^{2}+y^{2}+f(z, x)=0\right\} / \mathbb{Z}(0,1,1,1)$, әде $f(z, x)-\mathbb{Z}_{2}$-инвариант;

(d) $X \simeq\{\varphi(t, y, z, x)=0\} / \mathbb{Z}_{3}(1,2,2,0)$, әде $\varphi$ имеет вид 
(i) $\varphi=t^{2}+x^{3}+y z(y+z)$;

(ii) $\varphi=t^{2}+x^{3}+y z^{2}+x y^{4} \lambda\left(y^{3}\right)+y^{6} \mu\left(y^{3}\right)$, əде $4 \lambda^{3}+27 \mu^{2} \neq 0$;

(iii) $\varphi=t^{2}+x^{3}+y^{3}+x y z^{3} \alpha\left(z^{3}\right)+x z^{4} \beta\left(z^{3}\right)+y z^{5} \gamma\left(z^{3}\right)+z^{6} \delta\left(z^{3}\right)$;

(e) $X \simeq\{\varphi(t, y, z, x)=0\} / \mathbb{Z}_{2}(1,1,0,1)$, әде $\varphi$ имеет вид

(i) $\varphi=t^{2}+x y z+x^{2 a}+y^{2 b}+z^{c}$, где $a, b \geqslant 2, c \geqslant 3$;

(ii) $\varphi=t^{2}+y^{2} z+\lambda y x^{2 a+1}+g(x, z)$, əде $a \geqslant 1, \lambda \in \mathbb{C}, g(x, z) \in\left(x^{4}, x^{2} z^{2}, z^{3}\right)$. $\mathbb{C}\{x, z\}$

(f) $X \simeq\left\{t^{2}+x^{3}+g(y, z) x+h(y, z)=0\right\} / \mathbb{Z}_{2}(0,1,1,1)$, əде $g(y, z) \in(y, z)^{4} \mathbb{C}\{y, z\}$, $h(y, z) \in(y, z)^{4} \mathbb{C}\{y, z\} /(y, z)^{5} \mathbb{C}\{y, z\}$.

ОПРЕДЕлЕНИЕ 1.4. Пусть $X$ - нормальное логканоническое алгебраическое многообразие, и пусть $f: Y \rightarrow X$ - некоторое его раздутие. Предположим, что исключительное множество $f$ состоит только из одного неприводимого дивизора $E($ т.е. $\operatorname{Exc}(f)=E)$. Тогда $f:(Y, E) \rightarrow X$ называется чисто логтерминальным раздутием, если дивизор $K_{Y}+E$ чисто логтерминален и $-E f$-обилен.

ЗАмечАниЕ 1.5. В определении 1.4 неявно требуется, чтобы дивизор $E$ был $\mathbb{Q}$-Картье. Отсюда многообразие $Y$ имеет $\mathbb{Q}$-горенштейновые особенности.

ЗАмЕчАНИЕ 1.6. В обозначениях определения 1.4 имеем следующие свойства чисто логтерминальных раздутий.

1) Если многообразие $X$ имеет логтерминальные особенности, то дивизор $-\left(K_{Y}+E\right)$ $f$-обилен. Действительно, это следует из численной эквивалентности $K_{Y}+E \equiv$ $(a(E, 0)+1) E$ над $X$.

2) Если многообразие $X$ имеет строго логканонические особенности, то $a(E, 0)=-1$.

3) [8] Если многообразие $X$ является $\mathbb{Q}$-факториальным, то $Y$ тоже $\mathbb{Q}$-факториально и $\rho(Y / X)=1$. Отсюда следует, что в определении 1.4 для $\mathbb{Q}$-факториального случая, можно не требовать относительную обильность дивизора $-E$, так как она всегда имеет место. Напомним также, что каждая компонента исключительного множества любого бирационального стягивания в $\mathbb{Q}$-факториальную точку имеет коразмерность 1 .

4) [8] Пусть $f_{i}:\left(Y_{i}, E_{i}\right) \rightarrow(X \ni P), i=1,2,-$ два чисто логтерминальных раздутия. Если $E_{1}$ и $E_{2}$ определяют одно и то же дискретное нормирование поля функций $\mathscr{K}(X)$, то раздутия $f_{1}$ и $f_{2}$ изоморфны.

ОПРЕДЕЛЕНИЕ 1.7. Рассмотрим циклическую факторособенность

$$
Y:=\mathbb{C}^{n} / \mathbb{Z}_{m}\left(a_{1}, \ldots, a_{n}\right),
$$

где $a_{i} \in \mathbb{N}$ и $\left(a_{1}, \ldots, a_{n}\right)=1$ (случай $m=1$, т.е. $Y \simeq \mathbb{C}^{n}$, также возможен). Пусть $x_{1}, \ldots, x_{n}$ - координаты в $\mathbb{C}^{n}$, собственные для $\mathbb{Z}_{m}$. Взвешенным раздутием $Y c$ весом $\sigma=a_{1}, \ldots, a_{n}$ назьвается проективньй бирациональньй морфизм $f: \bar{Y} \rightarrow Y$ такой, что $\bar{Y}$ покрывается аффинными картами $U_{1}, \ldots, U_{n}$, где

$$
U_{i}=\mathbb{C}_{\bar{y}_{1}, \ldots, \bar{y}_{n}}^{n} / \mathbb{Z}_{a_{i}}\left(-a_{1}, \ldots, \underset{\uparrow}{m}, \ldots,-a_{n}\right) .
$$

Координаты в $Y$ и в $U_{i}$ связаны соотношениями

$$
y_{i}=\bar{y}_{i}^{a_{i} / m}, \quad y_{j}=\bar{y}_{j} \bar{y}_{i}^{a_{j} / m}, \quad j \neq i .
$$


Исключительное множество $E$ морфизма $f$ является неприводимым дивизором, $E \simeq$ $\mathbb{P}\left(a_{1}, \ldots, a_{n}\right)$.

Рассмотрим гиперповерхностную особенность

$$
X:=\left\{\varphi\left(x_{1}, \ldots, x_{n}\right)=0\right\} \subset \mathbb{C}_{y_{1}, \ldots, y_{n}}^{n}
$$

Пусть $\bar{X}=\bar{\pi}^{-1} X-$ собственный прообраз $X$ при отображении $\bar{\pi}, \pi=\left.\bar{\pi}\right|_{\bar{X}}-$ ограничение $\bar{\pi}$. Тогда $\pi: \bar{X} \rightarrow X$ тоже назьвается взвешенным раздутием $c$ весом $\sigma$ или просто взвешенным раздутием, если из контекста ясно, о каком $\sigma$ идет речь.

Через $\sigma w t(x)$ (соответственно $\sigma w t(y), \sigma w t(z), \sigma w t(u))$ или, если это понятно из контекста, через $w t(x)$ и т.д. мы будем обозначать вес $x$ (соответственно $y, z, u$ ) при взвешенном раздутии $\sigma$ (см. [4]). Вес монома $f(x, y, z, u)=x^{\alpha_{1}} y^{\alpha_{2}} z^{\alpha_{3}} u^{\alpha_{4}}$ при взвешенном раздутии $\sigma$ будет

$$
\sigma w t(f)=\alpha_{1}(\sigma w t x)+\alpha_{2}(\sigma w t y)+\alpha_{3}(\sigma w t z)+\alpha_{4}(\sigma w t u)
$$

или

$$
w t(f)=\alpha_{1}(w t x)+\alpha_{2}(w t y)+\alpha_{3}(w t z)+\alpha_{4}(w t u) .
$$

Для любого многочлена $f \in \mathbb{C}(x, y, z, u)$ вес $\sigma w t(f)$ - минимальный из $\sigma$-весов мономов, входящих в $f$.

\section{2. Стягивания индекса 1}

В этом разделе мы исследуем свойства дивизоров Картье, которые могут быть стянуты, и объемлющих их трехмерных многообразий.

Теорема 2.1. Пусть имеется некоторое дивизориальное стягивание $f: X \rightarrow Z$ такое, что стягивается неприводимый приведенный дивизор Картье $S, \operatorname{dim} X=$ $\operatorname{dim} Z=3, K_{X}+S$ чисто логтерминально, $Z$-росток терминальной особенности $P \in Z$. Тогда

(1) $X$ имеет терминальные особенности;

(2) дискрепантность $S$ минимальна над $Z$;

(3) над $P \in Z$ существует единственный дивизор с минимальной дискрепантностью;

(4) если $Z$ горенштейново, то $X$ тоже горенштейново, т.е. особенности $X$ не хуже, чем $c D v$ точки.

ДоказАтельство. (1) Рассмотрим вложенное разрешение $g: Y \rightarrow X$. Имеем

$$
K_{Y}+\widetilde{S}=g^{*}\left(K_{X}+S\right)+\sum\left(a_{i}-r_{i}\right) E_{i},
$$

где $E_{i}-g$-исключительные дивизоры, $a_{i}-$ их дискрепантности, $r_{i}-$ кратности, с которьми вклеиваются $E_{i}$ к полному прообразу $S$. Так как $a_{i}>-1, r_{i} \geqslant 1$, а особенности $S$ не хуже, чем логтерминальные, то $S$ не проходит через логтерминальные и канонические особенности $X$.

(2) Имеем $K_{X}=f^{*} K_{Z}+a S$, где $a>0$, так как $Z$ терминально. Пусть существует дивизор $E$ с дискрепантностью $b<a$ над $Z$. Тогда существует морфизм $\pi: Y \rightarrow X \rightarrow Z$ 
такой, что $E$ реализован на $Y$. Имеем $b=b_{X}+a k>a$, где $k \in \mathbb{Z}$ - кратность $S$ в центре $E$ на $X, b_{X}>0$. Противоречие.

(3) Аналогично, пусть $E$ - еще один дивизор с дискрепантностью $b=a$. Реализовав $E$, получаем $b=b_{X}+a k>a$, так как $b_{X}>0$.

(4) Пусть на $X$ существует негоренштейнова точка $F$. Тогда, реализовав дивизор $E$ с минимальной дискрепантностью $1 / m$ (над $F$ ), получим, что над $Z$ дискрепантность $E$ будет равна $b=1 / m+a k$. Так как $a k \in \mathbb{Z}$, то $b \notin \mathbb{Z}$. Противоречие с горенштейновостью $Z$.

\section{3. Случай, когда $Z$ - росток горенштейновой точки}

3.1. $c A$ случай. Если $Z$ - росток $c A$ точки (сюда же входит неособый случай), то возможны следующие варианты троек $(S, Z, f)$ (так как $f$ будет всегда взвешенньм раздутием, мы будем писать веса этого раздутия):

(1) $\left(\mathbb{P}^{2}, \mathbb{C}^{3},(1,1,1)\right)$ - гладкий случай;

(2) $\left(\left(t y+z^{2}+x^{2}=0\right) \subset \mathbb{P}^{3},\left(t y+z^{2}+x^{2}=0\right) \subset \mathbb{C}^{4},(1,1,1,1)\right)$;

(3) $\left(\left(t y+z^{2}=0\right) \subset \mathbb{P}^{3},\left(t y+z^{2}+x^{n}=0\right) \subset \mathbb{C}^{4},(1,1,1,1)\right)-$ случай $n>2$.

Как видно из работы [5], в остальных случаях дивизор с минимальной дискрепантностью над $P \in Z$ не один, поэтому из теоремы 2.1 следует, что других вариантов нет.

3.2. $c D_{4}$ случай. Чтобы описать этот случай, нам понадобится классификация (с точностью до аналитического изоморфизма) нормальных форм кубических кривых в $\mathbb{P}^{2}$ (см. [11]).

Теорема А. Нормальные формы (относительно $G L_{3}$ ) кубических кривых в $\mathbb{P}^{2}$ таковы:

(1) $g_{3}=y^{3}$

(2) $g_{3}=z^{2} y$

(3) $g_{3}=z y(z+y)$;

(4) $g_{3}=x y z$

(5) $g_{3}=\left(x^{2}+y z\right) y$

(6) $g_{3}=\left(x^{2}+y z\right) x$

(7) $g_{3}=\left(y^{2} z+x^{3}\right)$;

(8) $g_{3}=\left(y^{2} z+x^{3}+x^{2} z\right)$;

(9) $g_{3}=\left(x^{3}+y^{3}+z^{3}\right)+a x y z, a^{3}+27 \neq 0$.

ЗАмЕчАниЕ 3.1. Напомним, что $Z$ задается уравнением

$$
\left(t^{2}+g_{3}(x, y, z)+g_{\geqslant 4}(x, y, z)=0\right) \subset \mathbb{C}^{4} .
$$

3.2.1. Формы (1) и (2). Эти случаи относятся к особенностям вида $c D_{n}$ для $n \geqslant 5$, который будет рассмотрен ниже (см. п. 3.3).

3.2.2. Формы (3), (4), (5) и (6). В этих случаях чисто логтерминальные раздутия индекса 1 невозможны. Действительно, раздуем $Z$ с весами $(2,1,1,1)$. Если $g_{3}$ имеет вид (3) или (4), то вклеятся три дивизора с дискрепантностью $2+1+1+1-3-1=1$. Если $g_{3}$ имеет вид (5) или (6), то вклеятся два дивизора с дискрепантностью $2+1+1+1-$ $3-1=1$. Из теоремы 2.1 следует отсутствие чисто логтерминальных раздутий индекса 1 в этих случаях. 
3.2.3. Форма (7). В этом случае чисто логтерминальных раздутий индекса 1 не существует. Действительно, раздуем $Z$ с весами $(2,1,1,1): h: \bar{X} \rightarrow Z$. Вклеится один дивизор с дискрепантностью $a=2+1+1+1-3-1=1$. Проверим, что особенности многообразия $\bar{X}$ будут терминальными. Многообразие $\bar{X}$ покрывается аффинными картами $U_{1}, U_{2}, U_{3}, U_{4}$ :

$$
\begin{aligned}
& U_{1}=\left\{t+y^{2} z+x^{3}+\cdots=0\right\} / \mathbb{Z}_{2}(1,1,1,1) \\
& U_{2}=\left\{t^{2} y+z+x^{3}+\cdots=0\right\} \subseteq \mathbb{C}^{4} \\
& U_{3}=\left\{t^{2} z+y^{2}+x^{3}+\cdots=0\right\} \subseteq \mathbb{C}^{4} \\
& U_{4}=\left\{t^{2} x+y^{2} z+1+\cdots=0\right\} \subseteq \mathbb{C}^{4}
\end{aligned}
$$

Особенность $\bar{X}$ находится только в карте $U_{3}$ (это $c D_{4}$ точка). Исключительньй дивизор $E$ морфизма $h$ задается уравнением $E \simeq\left(y^{2} z+x^{3}=0\right) \subset \mathbb{C}^{4}$. Эта поверхность имеет хуже, чем логтерминальные особенности. А так как других дивизоров с дискрепантностью 1 нет (это следует из того, что в картах $U_{1}, U_{2}, U_{4}$ многообразие неособо, а в карте $U_{3}$ особенность $c D v$ ), то из относительной антиобильности $E$ получаем, что чисто логтерминальных раздутий индекса 1 в этих случаях не существует (см. [12]).

3.2.4. Форма (8). В этом случае чисто логтерминальных раздутий индекса 1 не существует. Действительно, раздуем $Z$ с весами $(2,1,1,1): h: \bar{X} \rightarrow Z$. Вклеится один дивизор с дискрепантностью $a=2+1+1+1-3-1=1$. Проверим, что особенности многообразия $\bar{X}$ будут терминальными. Многообразие $\bar{X}$ покрывается аффинными картами $U_{1}, U_{2}, U_{3}, U_{4}$ :

$$
\begin{aligned}
& U_{1}=\left\{t+y^{2} z+x^{3}+x^{2} z+\cdots=0\right\} / \mathbb{Z}_{2}(1,1,1,1), \\
& U_{2}=\left\{t^{2} y+z+x^{3}+x^{2} z+\cdots=0\right\} \subseteq \mathbb{C}^{4}, \\
& U_{3}=\left\{t^{2} z+y^{2}+x^{3}+x^{2}+\cdots=0\right\} \subseteq \mathbb{C}^{4}, \\
& U_{4}=\left\{t^{2} x+y^{2} z+1+z+\cdots=0\right\} \subseteq \mathbb{C}^{4}
\end{aligned}
$$

Особенность $\bar{X}$ находиться только в карте $U_{3}$ (это $c A$ точка). Исключительный дивизор $E$ морфизма $h$ задается уравнением $E \simeq\left(y^{2} z+x^{3}=0\right) \subset \mathbb{C}^{4}$. Эта поверхность имеет хуже, чем логтерминальные особенности. А так как других дивизоров с дискрепантностью 1 нет (это следует из того, что в картах $U_{1}, U_{2}, U_{4}$ многообразие неособо, а в карте $U_{3}$ особенность $c D v$ ), то из относительной антиобильности $E$ получаем, что чисто логтерминальных раздутий индекса 1 в этих случаях не существует (см. [12]).

3.2.5. Форма (9). В этом случае чисто логтерминальных раздутий индекса 1 не существует. Действительно, раздуем $Z$ с весами $(2,1,1,1): h: \bar{X} \rightarrow Z$. Вклеится один дивизор с дискрепантностью $a=2+1+1+1-3-1=1$. Проверим, что особенности многообразия $\bar{X}$ будут терминальными. Многообразие $\bar{X}$ покрывается аффинными картами $U_{1}, U_{2}, U_{3}, U_{4}$ :

$$
\begin{aligned}
& U_{1}=\left\{t+y^{3}+x^{3}+z^{3}+a x y z+\cdots=0\right\} / \mathbb{Z}_{2}(1,1,1,1) \\
& U_{2}=\left\{t^{2} y+z^{3}+x^{3}+1+a x z+\cdots=0\right\} \subseteq \mathbb{C}^{4} \\
& U_{3}=\left\{t^{2} z+y^{3}+x^{3}+1+a x y+\cdots=0\right\} \subseteq \mathbb{C}^{4} \\
& U_{4}=\left\{t^{2} x+y^{3}+1+z^{3}+a y z+\cdots=0\right\} \subseteq \mathbb{C}^{4}
\end{aligned}
$$


Многообразие $\bar{X}$ неособо. Исключительньй дивизор $E$ морфизма $h$ задается уравнением $E \simeq\left(x^{3}+y^{3}+z^{3}+a x y z=0\right) \subset \mathbb{C}^{4}$. Эта поверхность имеет хуже, чем логтерминальные, особенности. А так как других дивизоров с дискрепантностью 1 нет, то из относительной антиобильности $E$ получаем, что чисто логтерминальных раздутий индекса 1 в этих случаях не существует (см. [12]).

3.3. $c D_{n}$ случай для $n \geqslant 5$. В этом случае чисто логтерминальных раздутий индекса 1 нет. Действительно, рассмотрим раздутие $f: \bar{X} \rightarrow Z$ с весами $(w t(t), w t(y)$, $w t(z), w t(x))=(2,2,1,1)$. Многообразие $\bar{X}$ покрывается четырьмя аффинными картами $U_{1}, U_{2}, U_{3}, U_{4}$, где $U_{i}, i=1, \ldots, 4,-$ гиперповерхности в $\mathbb{C}^{4}$, заданные соответственно уравнениями

$$
\begin{aligned}
& U_{1}=\left\{1+y z^{2}+\cdots=0\right\} / \mathbb{Z}_{2}(1,0,1,1), \\
& U_{2}=\left\{t^{2}+z^{2}+\cdots=0\right\} / \mathbb{Z}_{2}(0,1,1,1), \\
& U_{3}=\left\{t^{2}+y+\cdots=0\right\} / \subseteq \mathbb{C}^{4} \\
& U_{4}=\left\{t^{2}+y z^{2}+\cdots=0\right\} \subseteq \mathbb{C}^{4}
\end{aligned}
$$

При этом исключительньй дивизор $E$ морфизма $f$ имеет дискрепантность 1 и задается уравнением

$$
E=\left\{t^{2}+y z^{2}+g_{w t=4}(x, y, z)=0\right\} \subseteq \mathbb{P}(2,2,1,1) .
$$

Нетрудно заметить, что в карте $U_{2}$ особенность типа $c A x / 2$. Раздуем взвешенно $\bar{Y} \rightarrow \bar{X}$ эту особенность с весами $\frac{1}{2}(2,1,1,1)$. Исключительный дивизор этого морфизма будет иметь дискрепантность (над $Z$ )

$$
a\left(Z, E_{1}\right)=1+\frac{1}{2}+\frac{1}{2}+\frac{1}{2}-1-1+\frac{1}{2}=1,
$$

где дискрепантность $a\left(\bar{Y}, E_{1}\right)=1 / 2$, а к дивизору $E$ вклеивается еще $\frac{1}{2} E_{1}$, так как $E$ локально задается уравнением $y=0$ вблизи особенности. Из теоремы 2.1 следует отсутствие чисто логтерминальных раздутий в этом случае.

3.4. $c E n$ случай. Как показано в работе [13], $Z$ имеет тип $c E n$ тогда и только тогда, когда

$$
Z \simeq\left(t^{2}+y^{3}+a z^{4}+b y z^{3}+c z^{5}+g_{\geqslant 6}(x, y, z)=0\right) \subset \mathbb{C}^{4}
$$

где хотя бы одно из чисел $a, b, c$ отлично от нуля. Рассмотрим следующие три случая.

Случай $a \neq 0$. Покажем, что в этом случае допустимых раздутий нет. Действительно, рассмотрим раздутие $f: \bar{X} \rightarrow Z$ с весами $(w t(t), w t(y), w t(z), w t(x))=(3,2,2,1)$. Многообразие $\bar{X}$ покрывается четырьмя аффинными картами $U_{1}, U_{2}, U_{3}, U_{4}$, где $U_{i}$, $i=1, \ldots, 4,-$ гиперповерхности в $\mathbb{C}^{4}$, заданные уравнениями

$$
\begin{aligned}
& U_{1}=\{1+\cdots=0\} / \mathbb{Z}_{3}(1,-2,-2,-1), \\
& U_{2}=\left\{t^{2}+1+\cdots=0\right\} / \mathbb{Z}_{2}(1,1,0,1), \\
& U_{3}=\left\{t^{2}+y^{3}+z^{2}+\cdots=0\right\} / \mathbb{Z}_{2}(1,0,1,1), \\
& U_{4}=\left\{t^{2}+y^{3}+\cdots=0\right\} \subseteq \mathbb{C}^{4} .
\end{aligned}
$$


При этом исключительньй дивизор $E$ морфизма $f$ имеет дискрепантность 1 и задается уравнением

$$
E=\left\{t^{2}+y^{3}+g_{w t=6}(x, y, z)=0\right\} \subseteq \mathbb{P}(3,2,2,1) .
$$

Нетрудно заметить, что в карте $U_{3}$ особенность типа $c A / 2$, которая нам дает еще, как минимум, один дивизор с дискрепантностью 1 (над $Z$ ). Из теоремы 2.1 следует отсутствие чисто логтерминальных раздутий в этом случае.

Случай $a=0, b \neq 0$. Покажем, что в этом случае допустимых раздутий нет. Действительно, рассмотрим раздутие $f: \bar{X} \rightarrow Z$ с весами $(w t(t), w t(y), w t(z), w t(x))=(3,2$, $2,1)$. Многообразие $\bar{X}$ покрьвается четырьмя аффинными картами $U_{1}, U_{2}, U_{3}, U_{4}$, где $U_{i}, i=1, \ldots, 4,-$ гиперповерхности в $\mathbb{C}^{4}$, заданные уравнениями

$$
\begin{aligned}
& U_{1}=\{1+\cdots=0\} / \mathbb{Z}_{3}(1,-2,-2,-1), \\
& U_{2}=\left\{t^{2}+1+\cdots=0\right\} / \mathbb{Z}_{2}(1,1,0,1), \\
& U_{3}=\left\{t^{2}+y^{3}+y+\cdots=0\right\} / \mathbb{Z}_{2}(1,0,1,1), \\
& U_{4}=\left\{t^{2}+y^{3}+\cdots=0\right\} \subseteq \mathbb{C}^{4} .
\end{aligned}
$$

При этом исключительньй дивизор $E$ морфизма $f$ имеет дискрепантность 1 и задается уравнением

$$
E=\left\{t^{2}+y^{3}+y z^{3}+g_{w t=6}(x, y, z)=0\right\} \subseteq \mathbb{P}(3,2,2,1) .
$$

Нетрудно заметить, что в карте $U_{3}$ особенность типа $\frac{1}{2}(1,1,1)$, которая нам дает еще один дивизор с дискрепантностью 1 (над $Z$ ). Из теоремы 2.1 следует отсутствие чисто логтерминальных раздутий в этом случае.

Случай $a=b=0, c \neq 0$. В этом случае допустимых раздутий нет. Действительно, рассмотрим раздутие $f: \bar{X} \rightarrow Z$ с весами $(w t(t), w t(y), w t(z), w t(x))=(3,2,2,1)$. Многообразие $\bar{X}$ покрывается четырьмя аффинными картами $U_{1}, U_{2}, U_{3}, U_{4}$, где $U_{i}, i=$ $1, \ldots, 4,-$ гиперповерхности в $\mathbb{C}^{4}$, заданные уравнениями

$$
\begin{aligned}
& U_{1}=\{1+\cdots=0\} / \mathbb{Z}_{3}(1,-2,-2,-1), \\
& U_{2}=\left\{t^{2}+1+\cdots=0\right\} / \mathbb{Z}_{2}(1,1,0,1), \\
& U_{3}=\left\{t^{2}+y^{3}+z^{4}+\cdots=0\right\} / \mathbb{Z}_{2}(1,0,1,1), \\
& U_{4}=\left\{t^{2}+y^{3}+\cdots=0\right\} \subseteq \mathbb{C}^{4} .
\end{aligned}
$$

При этом исключительньй дивизор $E$ морфизма $f$ имеет дискрепантность 1 и задается уравнением

$$
E=\left\{t^{2}+y^{3}+z^{5}+g_{w t=6}(x, y, z)=0\right\} \subseteq \mathbb{P}(3,2,2,1) .
$$

Покажем, что особенность в карте $U_{3}$ дает еще, как минимум, один дивизор с дискрепантностью 1 (над $Z$ ). Раздуем начало координат этой карты с весами $\frac{1}{2}(1,2,1,1)$ : $g: \bar{Y} \rightarrow \bar{X}$. Вклеится дивизор $E_{1}$ с дискрепантностью

$$
a\left(E_{1}, \bar{Y}\right)=\frac{1}{2}+1+\frac{1}{2}+\frac{1}{2}-1-1=\frac{1}{2} .
$$

А дискрепантность $a\left(E_{1}, Z\right)=a\left(E_{1}, \bar{Y}\right)+k(E)$. Так как $E$ задается $z=0$, а вес $z$ равен $1 / 2$, то $k(E)=1 / 2$. Поэтому $a\left(E_{1}, Z\right)=1$ и из теоремы 2.1 следует отсутствие чисто логтерминальных раздутий в этом случае. 


\section{4. Случай, когда $Z$ - росток негоренштейновой точки}

4.1. $c A / m$ случай. В случае циклической факторособенности мы имеем один допустимьй вариант:

(1) $\left(\mathbb{P}^{2}, \frac{1}{2}(1,1,1),(1,1,1)\right)$.

Это следует из терминальности $X$ и теоремы 2.1 .

В оставшихся случаях допустимых троек нет. Это следует из [4] и теоремы 2.1, так как в этих случаях число дивизоров с минимальной дискрепантностью больше 1.

4.2. $c A x / 2, c A x / 4, c D / 2, c D / 3$ и $c E / 2$ случаи. В этих случаях чисто логтерминальных раздутий индекса 1 нет. Действительно, в силу теоремы 2.1 мы можем рассматривать случаи, когда дивизор с минимальной дискрепантностью ровно один. Как видно из [4], [14], в терминальной категории нет раздутий, реализующих дивизор Картье с минимальной дискрепантностью. Из замечания $1.6(4)$ получаем, что в этих случаях допустимых раздутий нет.

\section{5. Случай, когда $Z-$ росток кривой}

5.1. Случай, когда $Z$ горенштейново. В этом случае $Z$ должно быть неособо, так как по теореме $2.1 X$ горенштейново, а над особенностью $Z$ существует единственный дивизор с минимальной дискрепантностью. Из раздела 3 и [15] следует, что если $Z$ особо, то стягиваемьй дивизор не является дивизором Картье. Из работы [16] следует, что допустимое чисто логтерминальное раздутие - это раздутие пучка идеалов приведенной неприводимой кривой, которая локально представляется как полное пересечение.

5.2. Случай, когда $Z$ негоренштейново. В этом случае чисто логтерминальных раздутий индекса 1 нет. Действительно, дискрепантность исключительной поверхности является целым числом и из теоремы 2.1 следует, что все дивизоры над $Z$ имеют дискрепантности $\geqslant 1$. Противоречие с [17], так как в разрешении терминальной особенности индекса $n$ должны присутствовать все дивизоры с дискрепантностью $i / n$.

Теорема 0.1 доказана.

\section{СПИСОК ЦИТИРОВАННОЙ ЛИТЕРАТУРЫ}

[1] Reid M. Young person's guide to canonical singularities // Proc. Symp. in Pure Math. 1987. V. 46. P. 343-416.

[2] Kawamata Y. Divisorial contractions to 3-dimentional terminal quotient singularities // Higher-Dimentional Complex Varieties (Trento, 1994). Berlin: Walter de Gruyter, 1996. P. 241-246.

[3] Kawakita M. General elements in anticanonical systems of threefolds with divisorial contractions and applications to classification // E-print math. AG/0110050, 2001.

[4] Hayakawa T. Blowing ups of 3-dimentional terminal singularities // RIMS Kyoto Univ. 1999. V. 35. P. 515-570.

[5] Фёдоров И. Ю. Раздутия трехмерньх терминальных особенностей: $c A$ случай // Матем. заметки. 2002. Т. 71. №3. С. 400-407.

[6] Shokurov V. V. Complements on surfaces. Algebraic geometry, 10 // J. Math. Sci. (New York). 2000. V. 102. № 2. P. 3876-3932.

[7] Шокуров В.В. Трехмерные логперестройки // Изв. РАН. Сер. матем. 1992. Т. 56. C. $105-203$. 
[8] Prokhorov Yu. G. Blow-ups of canonical singularities // Algebra / ed. Yu. Bahturin. Proc. Internat. Conf. on the Occasion of the 90th birthday of A. G. Kurosh (Moscow, Russia, May 25-30, 1998). Berlin: Walter de Gruyter, 2000. P. 301-317.

[9] Кудрявцев С. А. О чисто логтерминальных раздутиях // Матем. заметки. 2001. Т. 69. №6. C. 892-898.

[10] Kawamata Y., Matsuda K., Matsuki K. Introduction to the minimal model program // Algebraic Geometry. Sendai. Adv. Stud. Pure Math. 1987. V. 10. P. 283-360.

[11] Арнольд В.И., Варченко А. Н., Гусейн-Заде С. М. Особенности дифференцируемых отображений. М.: Наука, 1982.

[12] Kollar J. et al. Flips and Abundance for Algebraic Threefolds. Astérisque. V. 211. Paris: Soc. Math. France, 1992.

[13] Маркушевич Д. Г. Канонические особенности трехмерных гиперповерхностей // Изв. АН CCCP. Сер. матем. 1985. Т. 49. №2 2. С. 334-368.

[14] Hayakawa T. Blowing Ups of 3-dimentional Terminal Singularities, II // RIMS Kyoto Univ. 2000. V. 36. P. 423-456.

[15] Tziolas N. Terminal 3-fold divisorial contractions of a surface to a curve, I // E-print math.AG/0110074, 2001.

[16] Cutkosky S. Elementary contractions of Gorenstein threefolds // Math. Ann. 1988. V. 280. P. 521-525.

[17] Шокуров В.В. Добавление к статье "Трехмерные логперестройки" // Изв. РАН. Сер. матем. 1993. Т. 57. №6. С. 141-175.

Математический институт им. В. А. Стеклова РАН

Поступило

E-mail : ifedorov@mi.ras.ru 